\title{
Intervascular Line Infections: Can We Prevent Them? Bring on the Team!
}

\author{
Peter J. Papadakos ${ }^{*}$ (1) \\ (c) 2019 Springer Science+Business Media, LLC, part of Springer Nature and Neurocritical Care Society
}

Central venous catheters (CVCs) and peripherally inserted central catheters (PICCs) are essential tools in the care of critically ill intensive care unit (ICU) patients. They allow for administration of large amounts of intravenous fluids, hypertonic fluids and vasopressor agents. They are also important in monitoring central venous pressure and for the easy collection of laboratory samples. Despite the clear cut benefits of CVCs and PICCs, these lines are not without inherent risks.

These two types of catheters have different complication profiles [1, 2]. Several national organizations and regulatory agencies have focused on decreasing the complication rates of these catheters. Lately, investigators have focused on care bundles to provide guidance on the insertion practices and care of these lines. Units throughout the world are ranked based on their infection rates which are tabulated and also major quality measures as to the care of critically ill patients [3]. Herc et al. [4] developed a mode to predict central line-associated blood stream infections in PICCs and created a scoring system called the MPC score which identified a number of factors associated with adverse events. Two major factors are the number of lumens and the administration of total parenteral nutrition. However, they did not control for the training of individuals inserting the lines and the variability of practitioners caring for these lines.

Brandmeir et al. [5] took on this long standing, complicated issue in a neurointensive care unit in a large

\footnotetext{
*Correspondence: Peter_Papadakos@urmc.rochester.edu Division Critical Care Medicine, Departments of Anesthesiology and Perioperative Medicine, Surgery, Neurology and Neurosurgery, University of Rochester School of Medicine and Dentistry, 601 Elmwood Avenue, Box 604, Rochester, NY 14642, USA

This comment refers to the article available at https://doi.org/10.1007/ s12028-019-00843-Z.
}

academic center. They addressed the problem by developing a special team with the responsibility of placing the lines and who were also available 24-h per day. Their results are based on close follow-up of cultures to identify central line-associated systemic infection and venous Doppler studies to identify line venous thrombosis (LVT). The study showed a very low number of complications with the total number of complications between the two groups PICCs and CVCs, where similar. The PICC cohort suffered 4 LVTs and one insertion issue of pneumothorax in the CVC group. The major point of this study is that with a highly trained, limited group of motivated practitioners inserting all CVC and PICC lines $24 / 7$, you can greatly reduce the majority of problems within both types of lines and thus erase the differences found in other studies.

The strength of this study also limits its transfer to other units across the globe. Because the reality of critical care is that it is a 24/7 practice, many individuals place lines in our units; nursing, advanced practice providers residents, fellows, attendings and even students, all often place lines in both routine and emergent situations. I cannot see a practitioner needing to administer $23 \%$ hypertonic saline to a critically ill patient, waiting for a specially trained individual on a central line team to come in from home at $3 \mathrm{am}$. But having said this, I do believe several highly important findings can be transferable to all ICUs. First and foremost, a highly structured program needs to be implemented in all facilities to train and certify individuals responsible for line insertion. This should be done and supervised by a small cadre of individuals with an ongoing observation program to assess the certified individuals from time to time, in order to prevent natural variability from occurring. What staff is certified and trained to place these central lines should be developed by each facility based on which professionals are authorized to do these procedures based on state and

\section{Springer}


national licensing requirements. The training program should include both classroom training and supervised hands-on training at the bedside. What the number of lines to be done under supervision prior to the individual being "certified" should be decided by each individual institution or health system, I believe 25 may be a good goal post in that many fellowship programs are currently using this number for multiple other procedures. The time-out tool used in operating rooms by physicians and nurses is a prime example of how education and standardization can easily decrease errors and increase positive patient outcomes.

This paper is also a call that every unit develop a select group of staff to care for and teach individuals how to properly access central lines. The primary cause for many line infections is improper non-sterile access of the line. Such clinician experts can be key resource educators to orient staff both on the floors and in the ICUs that care for these lines. Also every staff member should be empowered to stop an individual who is improperly accessing the line and correct an improper action. The national "Hand Wash Hand" initiative is a great example how such staff empowerment can decrease hospital infections. This program can be reinforced with posters and educational videos located in staff lounges. Utilization of the robust electronic medical record can also be used as a key tool to prevent central line complications. One of the most important factors to decrease complications from both CVCs and PICC lines is to remove them when no longer needed. The inclusion of alerts in the electronic medical record can be essential to alert providers when lines have been in for extended periods of time. Care givers should be asked each day whether the line is necessary today.
These simple points can easily be implemented to address an issue of complications such as central line and PICC infections and thrombus that has plagued hospitals and ICUs since the introduction of central venous access. Sometimes a study that controls the most important variable, individual practices, answers many questions.

Conflict of interest

The author declares that he has no conflict of interest.

\section{Publisher's Note}

Springer Nature remains neutral with regard to jurisdictional claims in published maps and institutional affiliations.

Published online: 27 September 2019

\section{References}

1. Chopra V, Anand S, Krein SL, Cenoweth C, Saint S. Bloodstream infection, venous thrombosis and peripherally inserted central catheters: reappraising the evidence. Am J Med. 2012;125:733-41.

2. Bell T, O'Grady N. Prevention of central line-associated bloodstream infections. Infect Dis Clin. 2017;31(3):551-9.

3. Payne V, Hall M, Prieto J. Johnson M Care bundles to reduce central line-associated bloodstream infections in the neonatal unit: a systematic review and meta-analysis. Arch Dis Child Fetal Neonatal Ed. 2018;108:F422-9.

4. Herc E, Patel P, Washer LL, et al. A model to predict central-line-associated bloodstream infection among patients with peripherally inserted central catheters: the MPC score. Infect Control Hosp Epidemiol. 2017;38:1155-66.

5. Brandmeir NJ, Davanzo JR, Payne R, et al. A randomized trial of complications of peripherally inserted central lines and centrally inserted central lines in the neuro-intensive car unit. Results of the NSPVC Trial Neurocrit Care 2019. 